\title{
Possible bridging the classical physics and quantum physics through consciousness
}

\author{
Dhananjay Pal \\ Email address: \\ dhananjay.pal123@gmail.com,paldhananjay46@yahoo.com
}

Pharmacy College, Bengal School of Technology, Sugandha-Delhi Road, Chuchura, Dist.-Hooghly, West Bengal, INDIA

\section{To cite this article:}

Dhananjay Pal. Possible Bridging the Classical Physics and Quantum Physics through Consciousness. American Journal of Modern Physicy. Vol. 2, No. 6, 2013, pp. 322-329. doi: 10.11648/j.ajmp.20130206.18

\begin{abstract}
Scientists have arrived at a simple but decisive conclusion that consciousness is very much a part of the universe, like other objects. Our consciousness model involving thought-carrying particle (TCP), thought retaining particle (TRP) and thought force $\left(\mathrm{T}_{\mathrm{F}}\right)$ signifies the existence of universal consciousness that exists along with the universe. This universal consciousness is a functional state of the universal mind. This universal mind (UM) is evolved at the Big Bang from void. The UM is constituted by these TCP and TRP in the inherent presence of thought force $\left(T_{F}\right)$. Thought force $\left(T_{F}\right)$ is an expression of universal consciousness. A single field emerged at the origin of the universe, already containing within itself the blueprint of the physical universe. The primordial single field triggered the onset of the universe. Most physicists believe that a single super-force dominated the first instants of creation. The Thought force $\left(\mathrm{T}_{\mathrm{F}}\right)$ being the primordial quantum field functions as the original super-force. $\mathrm{T}_{\mathrm{F}}$ being the original super-force functions as the origin of all the fundamental fields. TCP is the carrier of thought force $\left(\mathrm{T}_{\mathrm{F}}\right)$ that, in turn, appears to be the origin of all the fields. The quantized energy $\left(\varepsilon_{T}\right)$ of TCP is responsible to cause the universal consciousness as well as the cosmic microwave background radiation temperature. The individual consciousness owes its origin to the universal consciousness created by the same $\varepsilon_{T}$. The same $\varepsilon_{T}$ is the energy responsible for generating thought force $\left(\mathrm{T}_{\mathrm{F}}\right)$. $\mathrm{T}_{\mathrm{F}}$ being an expression of the universal consciousness is applicable to any inanimate object as well as to any biological system (having thinking ability). The $\mathrm{T}_{\mathrm{F}}$ exerts its functions both in vitro and in vivo. This $\varepsilon_{T}$ (i.e. universal consciousness) is found to form a possible gross bridge between the classical physics and quantum physics.
\end{abstract}

Keywords: Universal Mind (UM), Thought Force $\left(\mathrm{T}_{\mathrm{F}}\right), \mathrm{T}_{\mathrm{F}}$ (Micro), $\mathrm{T}_{\mathrm{F}}$ (Macro), Though-Carrying Particle (TCP), Thought Retaining Particle (TRP), Quantized Energy $\left(\boldsymbol{\varepsilon}_{T}\right)$ of TCP.

\section{Introduction}

It is most relevant and important to indicate the names of various eminent physicists like Erwin Schrödinger, Eugene Wigner, Brian Josephson, John Wheeler, Roger Penrose, Henry P Stapp, Freeman J. Dyson, Paul Davies, David Bohm, Basil Hiley, Fritjof Capra, Fred Alan Wolf and Amit Goswami who have addressed the inclusion of consciousness in their work. Consciousness is to be taken into account. The consciousness model of Pal et al [1-3] incorporating TCP, TRP and thought force $\left(\mathrm{T}_{\mathrm{F}}\right)$ signifies the existence of universal consciousness that exists along with the universe.

\subsection{General View for the Evolution of the Universe}

Big Bang $\rightarrow$ Universe $\rightarrow$ [Fields + Particles \&

Antiparticles + STC $] \rightarrow[$ Matter and Fields + STC $] \rightarrow$ Matter and Fields + STC + Life as well as consciousness.

If the universe exists along with the universal consciousness that, in turn, is a functional state of the universal mind (UM) and if this UM is evolved at the Big Bang from the eternal void, then

\section{Our view for the evolution of the universe (see Scheme-I)}

VOID $\rightarrow$ [Big Bang $] \rightarrow[$ TCP, TRP \& Anti-TRP in the inherent presence of thought force $\left.\left(\mathrm{T}_{\mathrm{F}}\right)+\mathrm{STC}\right] \rightarrow$ [Universal Mind with universal consciousness + TCP \& TRP in the inherent presence of thought force $\left(\mathrm{T}_{\mathrm{F}}\right)+\mathrm{STC}+$ $\mathrm{UTF}] \rightarrow$ [Universal Mind with universal consciousness + Matter and Fields \{including thought force $\left.\left(\mathrm{T}_{\mathrm{F}}\right)\right\}+$ TCP \& $\mathrm{TRP}+\mathrm{STC}+\mathrm{UTF}] \rightarrow$ Universal Mind with universal consciousness + Matter and Fields \{including thought force $\left.\left(\mathrm{T}_{\mathrm{F}}\right)\right\}+$ Life as well as consciousness $+\mathrm{TCP} \& \mathrm{TRP}+\mathrm{STC}$ + UTF [where Thought force $\left(\mathrm{T}_{\mathrm{F}}\right)$ is the origin of all the 
fields, TCP (Though-carrying particle) is the origin of all the field particles, TRP (Thought retaining particle) is the origin of all the matter particles, anti-TRP is the origin of all the anti-particles, STC $=$ Space time continuum and $\mathrm{UTF}=$ Universal thought frequency $\cong$ frequency of the TCP].

Bhaumik [4] mentioned, "It would be reasonable to presume that the universe originated in a unity of all fields at or near Planck's dimension. John Wheeler strongly believes that "in defining any useful concept of reality" we have to take into account "the indispensable place of the participating observer---evidenced in quantum mechanics," the foregoing cannot be really true unless consciousness is as essential as aspect of nature as are the fields that give rise to force and matter and the primary field that gave rise to them". Consciousness is to be taken into account.

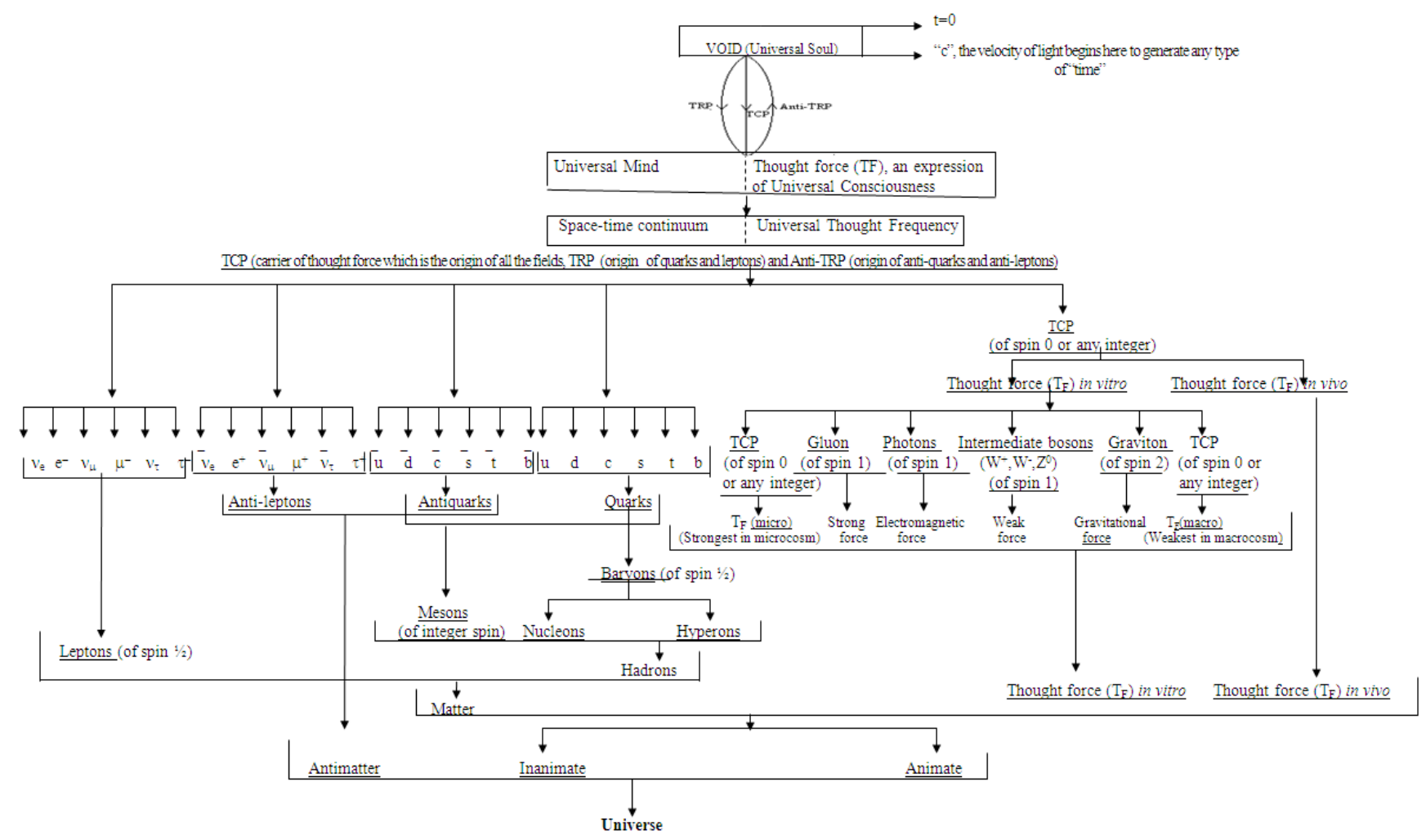

Scheme-I: Schematic presentation showing the evolution of the universe from the Void incorporating the postulated TCP (Thought-carrying particle), TRP (Thought-retaining particle) and Thought Force $\left(T_{F}\right)$. Laws of absolute conservation of mass, charge (and color) are to be maintained in order to develop a general theory for the unification of physics which would be freely applicable to the more general situations involving both the non-living system and living system having consciousness. Here TCP is the carrier of thought force $\left(T_{F}\right)$ that is the origin of all the fields. TRP is the origin of all the matter particles. It is to be noted that these TCP and TRP function like wavicle: wave-particle duality. Here thought force ( $T_{F}$ ) is an expression of the universal consciousness.

1.2. Consciousness model of Pal et al [1-3] involving TCP, TRP and thought force $\left(\mathrm{T}_{\mathrm{F}}\right)$ signifies the existence of universal consciousness that exists along with the universe. Pal et al [3] showed that the functional state of Universal Mind (UM) is the universal consciousness that exists along with the universe. Pal et al [3] explained that the UM is evolved at the Big Bang from the eternal Void (see Scheme-I). This Void, in turn, is the source of infinite energy. And this UM is a finer matter. The individual mind being a constituent of the UM is also a finer matter. The constituents of the UM and individual mind are the same. The ultimate constituents of matter and mind are the same as both mind and matter are aspects of one fundamental reality, which is called UM. The brain is the mediating link or interface between the individual mind and body.

Pal et al [3] explained that the constituents of the UM are the ultimate constituents of matter itself as everything in this universe is a manifestation of this UM. Pal et al [3] expressed that the UM is constituted by these TCP and TRP in the inherent presence of thought force $\left(\mathrm{T}_{\mathrm{F}}\right)$. Pal et al $[1,3]$ further explained that the ultimate constituents of matter and mind are these TCP and TRP in the inherent presence of thought force $\left(\mathrm{T}_{\mathrm{F}}\right)$ in vitro and thought force $\left(\mathrm{T}_{\mathrm{F}}\right)$ in vivo [See Scheme-I].

Pal et al [1,3] further explained that the existence of universal consciousness is the primary cause for the evolution of life. Without the inherent existence of universal consciousness, the inanimate matter itself cannot generate life and consciousness. These TCP and TRP, the constituents of the UM and the ultimate constituents of matter and mind are conceived here to be originated from the Void at the Big Bang to evolve the 'spacetime continuum' and the UM along with the universal consciousness. Thought force $\left(\mathrm{T}_{\mathrm{F}}\right)$, an expression of this universal consciousness, is thus the primordial quantum field and it exerts its functions both in vitro and in vivo (see Scheme-I).

Physicists determined that underlying quantum fields 
give birth to elementary particles. Bhaumik [4] mentioned that Frank Wilczek pointed out, "In quantum field theory, the primary elements of reality are not individual particles, but underlying fields. Thus, for example, all electrons are but excitations of an underlying field, naturally called electric field". The same holds true for all the fundamental particles of which matter is made.

The existence of matter depends on the existence of force and vice versa. TCP cannot exist without TRP and vice versa. Many physicists believe that unifying all the forces, including gravity, into a single theory would require a phenomenon called super-symmetry. With supersymmetry, every fermion would have a boson twin, and vice-versa. The thought force $\left(\mathrm{T}_{\mathrm{F}}\right)$ is carried by the TCP in the presence of its super-symmetrical partner TRP. TCP that behaves like boson should accompany its supersymmetrical partner TRP that functions like fermion in the generalized simpler way. It is to be noted that these TCP and TRP function like wavicle: wave-particle duality.

1.3. Pal et al [1-3] showed that the quantized energy $\left(\varepsilon_{T}\right)$ of TCP is responsible to cause the universal consciousness as well as the cosmic microwave background radiation temperature. Here thought force $\left(\mathrm{T}_{\mathrm{F}}\right)$ is an expression of the universal consciousness which exists along with the universe. And the quantized energy $\left(\mathcal{E}_{T}\right)$ of TCP represents universal consciousness.

1.4. Pal et al [3] developed three different equations expressing the quantized energy ( $\boldsymbol{\varepsilon}_{T}$ ) of TCP radiated from the radiant mass of universe. The value of $\varepsilon_{T}$ in one of the three equations is shown below:

$$
\begin{aligned}
& \mathcal{E}_{T} \\
= & 4.384 \times 10^{-16} \mathrm{erg} \equiv 2.73 \times 10^{-4} \mathrm{eV} \equiv 2.73^{0} \mathrm{~K} \cong C M B R \text { temperature } \\
\equiv & 2.725^{0} \mathrm{~K}
\end{aligned}
$$$$
\text { It is indicated by Weisskopf [5] that }
$$$$
1 e r g \cong 0.6241807 \times 10^{12} \mathrm{eV} \text { and } 10^{-4} \mathrm{eV} \cong 1^{0} \mathrm{~K} \text { where } \mathrm{K}=
$$
Kelvin.

1.5. Pal et al [3] expressed that the cosmic microwave background radiation (CMBR) temperature is due to the $\varepsilon_{T}$, the quantized energy of TCP where $\varepsilon_{T}=4.384 \times 10^{-16} \mathrm{erg} \equiv 2.73^{\mathrm{o}} \mathrm{K}$ that is very close to $2.725^{\circ} \mathrm{K}$ (CMBR temperature). The CMBR temperature exists due to the existence of $\varepsilon_{T}$ of the TCP radiated from the radiant mass of universe. As per Pal et al [3], it is interesting to note that all of the three different equations ultimately give rise to the same result that is equivalent to the CMBR temperature. A sort of relationship is thus observed between the $\varepsilon_{T}$ and the CMBR temperature. This coincidence thus signifies a probable role of TCP on the maintenance of CMBR temperature. Further, this coincidence also signifies the existence of these TCP in the presence of TRP. TCP cannot exist without TRP and vice versa. The presence of TCP, TRP and Thought force $\left(\mathrm{T}_{\mathrm{F}}\right)$ in the universe is thus indicated and expressed mathematically. At present we are unable to explain when and how these
TCP and TRP decoupled from the primordial cosmic soup.

\section{Thought Force}

Pal [6] explained the existence of thought force. A single field emerged at the origin of the universe, already containing within itself the blueprint of the physical universe. The primordial single field triggered the onset of the universe. Most physicists believe that a single superforce dominated the first instants of creation. This mysterious super-force is the Thought force $\left(T_{F}\right)$ in our view. It is possible that the evolution of all the fundamental fields is originated from this super-force that is nothing but the Thought force $\left(\mathrm{T}_{\mathrm{F}}\right)$ itself. Pal [6] explained that Thought force $\left(\mathrm{T}_{\mathrm{F}}\right)$, an expression of the universal consciousness, is the primordial quantum field that, in turn, functions as the primary unified field. Thought force $\left(T_{F}\right)$ itself may thus be found to be the original single primary unified field that is the origin of all the four fundamental fields along with both the $T_{F}$ (micro) and $T_{F}$ (macro). $\mathrm{T}_{\mathrm{F}}$ being an expression of the universal consciousness is applicable to any inanimate object as well as to any biological system (having thinking ability). The $\mathrm{T}_{\mathrm{F}}$ exerts its functions both in vitro and in vivo.

Pal [6] explained that the non-living system of the world is governed by the thought force $\left(\mathrm{T}_{\mathrm{F}}\right)$ in vitro and this Thought force $\left(\mathrm{T}_{\mathrm{F}}\right)$ in vitro gives rise to $\mathrm{T}_{\mathrm{F}}$ (micro), $\mathrm{SNF}$, EMF, WNF, GF and $\mathrm{T}_{\mathrm{F}}$ (macro)

where $\mathrm{T}_{\mathrm{F}}($ micro $)=$ Thought force in microcosm, $\mathrm{SNF}=$ Strong nuclear force, $\mathrm{EMF}=$ Electromagnetic force, $\mathrm{WNF}=$ Weak nuclear force, $\mathrm{GF}=$ Gravitational force and $\mathrm{T}_{\mathrm{F}}$ (macro) $=$ Thought force in macrocosm. It is to be noted here that $\mathrm{T}_{\mathrm{F}}$ (micro) is a stronger force than the SNF and $\mathrm{T}_{\mathrm{F}}$ (macro) is a weaker force even than the GF.

Pal [6] expressed that the living system of the world is governed by the thought force $\left(\mathrm{T}_{\mathrm{F}}\right)$ in vivo and this Thought force $\left(\mathrm{T}_{\mathrm{F}}\right)$ in vivo is a type of force that represents the biological 'thought' which is the action of mind. This 'thought' being a type of force controls the 'thought processes' involving the firing of neurons through the quantum mechanical activities of these postulated TCP and TRP in the presence of consciousness. This consciousness, in turn, is the quantized energy $\left(\varepsilon_{T}\right)$ of TCP.

\section{Classical Physics and Quantum Physics}

In order to understand the functions of the universe conspicuously, modern scientists divided the whole universe into two parts: (1) Macrocosm (large scale structure) and (2) Microcosm (tiny scale structure).

(1) Macrocosm (large scale structure) is governed by the classical physics that, in turn, follows

Einstein's Equation: $E=m c^{2}$ where $\mathrm{E}=$ energy in erg, $\mathrm{m}=$ mass in $\mathrm{gm}, \mathrm{c}=$ free-space velocity of light $=3 \times 10^{10}$ $\mathrm{cm} / \mathrm{sec}$. 
(2) Microcosm (tiny scale structure) is governed by the quantum physics (: the physical theory that deals with matter and energy in the smallest possible scale). It follows Planck's Equation: $E=h v=h c / \lambda$

where $\mathrm{E}=$ energy in erg,

$\mathrm{h}=$ Planck's constant $=6.63 \times 10^{-27}$ erg. sec,

$v=$ frequency in $\mathrm{Hz}$ or cps,

$\mathrm{c}=$ free-space velocity of light $=3 \times 10^{10} \mathrm{~cm} / \mathrm{sec}$,

$\lambda=$ wavelength in $\mathrm{cm}$.

It is to be noted that there is another equation of energy called Planck Energy $\left(E_{p}\right)$ which is expressed as follows:

$$
\text { Planck Energy }\left(E_{P}\right)=2 \pi\left(h c^{5} / G\right)^{1 / 2} \approx 3.09 \times 10^{17} \mathrm{erg}
$$

This $E_{p}$ (Planck energy) is always free as expressed by Wheeler $[7]$.

\section{What Is Life}

Life is a state of flux that is being maintained by a typical form of energy which is nothing but the consciousness itself. Pal et al [3] and Pal [8] showed that the quantized energy $\left(\varepsilon_{T}\right)$ of TCP is responsible to cause the universal consciousness as well as the cosmic microwave background radiation temperature. The individual consciousness owes its origin to the universal consciousness created by the same $\varepsilon_{T}$. Pal et al $[1,3]$ and $\mathrm{Pal}$ [8] explained that life may be defined as a state of functional manifestation of consciousness that, in turn, is the quantized energy ( $\varepsilon_{T}$ ) of TCP. This $\varepsilon_{T}$ represents universal consciousness.

Thus, Life $=\mathrm{f}($ Consciousness $)=\mathrm{f}\left(\mathcal{E}_{T}\right)$

where $\varepsilon_{T}=$ quantized energy of the TCP $=4.384 \times 10^{-16} \mathrm{erg}$.

$$
\varepsilon_{T}=h v_{T}=h c / \lambda_{T}=4.384 \times 10^{-16} \mathrm{erg}
$$

where $\varepsilon_{T}=$ quantized energy of the TCP

$=4.384 \times 10^{-16} \mathrm{erg}$,

$\boldsymbol{v}_{T}=$ frequency of the TCP

$=\varepsilon_{T} / h=66.12 \times 10^{9} \mathrm{~Hz} .=66.12 \mathrm{GHz}$,

$\mathrm{h}=$ Planck's quantum constant $=6.63 \times 10^{-27} \mathrm{erg} \cdot \mathrm{sec}$,

$\mathrm{c}=$ free-space velocity of light $=3 \times 10^{10} \mathrm{~cm} / \mathrm{sec}$,

$\lambda_{T}=$ wave-length of the $\mathrm{TCP}=0.4537 \mathrm{~cm}$.

\subsection{What Is Consciousness}

Psychologists, neuroscientists, philosophers, and other professionals continue to engage in an ongoing debate as to what consciousness means. In reality, we may never know.
Is it a product of the biological and classical physical interactions of the human brain; or is it something more fundamental, perhaps electromagnetic, or the result of quantum physics principles that we don't yet fully understand? Could it be something even more profound than that -- something beyond the scope of science and physics, any kind of physics, for us to understand?

According to the Vedanta, consciousness is not an emergent property of matter that comes into existence only through the functioning of the human nervous system. Instead, consciousness is a characteristic of reality, pervading all manifestations. This unbounded field of nature's 'universal consciousness' is not limited to an individual consciousness. From this viewpoint, the role of the human nervous system is to provide an appropriate material structure to individualize consciousness. It appears that inanimate matter itself cannot generate 'consciousness' without the inherent existence of 'universal consciousness'. Consciousness in living organisms is a process which involves the quantum mechanical activities of these TCP and TRP, the ultimate constituents of any matter as well as any mind in the inherent presence of thought force $\left(\mathrm{T}_{\mathrm{F}}\right)$ in vitro and the thought force $\left(\mathrm{T}_{\mathrm{F}}\right)$ in vivo as indicated by Pal et al [1,3]. And these TCP and TRP govern the activities of neurons (not the other way round). Neurons are simply the equipments used to generate consciousness and awareness.

Pal et al $[1,3]$ explained that the consciousness itself is functioning as an inter-linking agent between the animate and inanimate. The quantum mechanical activities of these TCP and TRP could also explain self-organization. Consciousness may thus be defined as the 'self-organized' capability of any living being to activate TCP and TRP, the ultimate constituents of mind and matter and to exert its functions.

Pal et al [2] explained, "Consciousness is the realization of existence, and there are as many states of consciousness as there are states of existence. Every living being has a consciousness of its own depending on complexity of the brain and the activity of the viable numbers of TCP in the presence of TRP; and the state of its consciousness changes every moment of time. Consciousness is the perception of the relation it bears to things and as this relation changes, consciousness changes its character. Consciousness itself does not change; it only moves up and down on the 'scale of the realization' of existence through the 'sub-conscious', 'conscious' and 'super-conscious' states'. There is another term called unconscious state. The most usual unconscious state is sleep. A deeper form of unconsciousness is called a coma.

It appears that

$$
\text { Consciousness }=\mathrm{f}(\text { mind })
$$

Consciousness is the functional state of mind. Presence of consciousness signifies the presence of mind and vice versa.

It is apparent that 


$$
\text { Animate } \neq \text { Inanimate }
$$

Three critical factors (which distinguish life from nonliving) are consciousness, metabolism and reproduction.

In a gross presentation, we can express from the equation (5):

$$
\text { Animate }- \text { Consciousness }=\text { Inanimate }
$$

Scientists would have to define and characterize consciousness properly. We should have the proper knowledge about the exact characteristics of consciousness in order to address many present day scientific enigmas.

\section{Possible Bridge between the Classical Physics and Quantum Physics through Consciousness $\left(=\varepsilon_{T}=\right.$ Quantized Energy of the TCP)}

The problem is the search for a theory that encompasses both the effects of gravity, described by Einstein's theory of general relativity, and the fuzziness that occurs in the realm of tiny particles according to quantum mechanics (: the physical theory that deals with matter and energy in the smallest possible scale). The scientific community is not yet able to explain why the micro world follows quantum rules and the macro one classical.

As per Hawking [9], the total energy of the universe is zero. Hawking [9] expressed that the negative gravitational energy exactly cancels the positive energy represented by the matter supposing the universe to be approximately uniform and smooth in space i.e.

$$
E_{T}=E_{M u}+\left(-E_{G u}\right)=0
$$

where $E_{T}=$ total energy of the universe,

$E_{M u}=$ positive energy represented by the total matter content of the universe,

$-E_{G u}=$ negative energy represented by the gravitational field of the universe.

It is to be noted that the quantized energy $\left(\varepsilon_{T}\right)$ of TCP exists along with the universe to cause the universal consciousness as well as CMBR temperature. In our proposition, the overall total energy $\left(E_{T}\right)$ of the universe is not zero but equals the specified quantized energy $\left(\varepsilon_{T}\right)$ of TCP that exists along with the universe i.e.

$$
E_{T}=E_{M u}+\left(-E_{G u}\right)+\varepsilon_{T}
$$

where $E_{T}=$ total energy of the universe,

$E_{M u}=$ positive energy represented by the total matter content of the universe,
$-E_{G u}=$ negative energy represented by the gravitational field of the universe,

$\boldsymbol{\varepsilon}_{T}=$ quantized energy of the TCP.

Therefore,

$$
E_{T}=\varepsilon_{T}=m_{T} V_{T} c \cong m_{T} c^{2}=h v_{T}
$$

$\left[\because V_{T}\right.$, the velocity of TCP $\cong \mathcal{C}$, the free-space velocity of light]

where $m_{T}=$ quantized mass of the TCP, $\mathrm{h}=$ Planck's quantum constant $=6.63 \times 10^{-27} \mathrm{erg} . \mathrm{sec}$, and

$\nu_{T}=$ frequency of the TCP.

At this juncture, it is provocative to note that this $\mathcal{E}_{T}$ may form a possible gross bridge between the classical physics and quantum physics through the existence of the expression:

$$
E_{T}=\varepsilon_{T}=m_{T} c^{2}=h v_{T}=h c / \lambda_{T}=4.384 \times 10^{-16} \mathrm{erg}
$$

where $E_{T}$ is the total energy of the universe. In this proposition, the overall total energy $\left(E_{T}\right)$ of the universe is not zero but equals the specified $\mathcal{E}_{T}$ as the universe exists along with the $\varepsilon_{T}$.

It is observed through the equation (10) that $\varepsilon_{T}$, the quantized energy of TCP is equal to $E_{T}$ where $E_{T}$ is the total energy of the universe. This $E_{T}$ obeys $E=m c^{2}$. It is to be noted that when $\varepsilon_{T}$ is equal to $E_{T}$, this $\varepsilon_{T}$ should also obey $E=m c^{2}$ apparently however odd it may appear. It is thus apparent that consciousness $\left(=\mathcal{E}_{T}\right)$ is forming a possible gross bridge between the classical physics and quantum physics and this specified $\varepsilon_{T}$ (= consciousness) is found to exist along with the universe as universal consciousness.

It is found that similar to the Einstein's equation: $E=m c^{2}$, consciousness can be expressed as

$$
\varepsilon_{T}=m_{T} c^{2}=4.384 \times 10^{-16} \mathrm{erg} \quad \text { (Classical physics) }
$$

where $\varepsilon_{T}=$ quantized energy of the TCP $=4.384 \times 10^{-16} \mathrm{erg}$,

$m_{T}=$ quantized mass of the TCP $=4.87 \times 10^{-37} \mathrm{~g}$ and

$\mathrm{c}=$ free-space velocity of light $=3 \times 10^{10} \mathrm{~cm} / \mathrm{sec}$.

It is also found that similar to Planck's Equation: $E=h v=h c / \lambda$, consciousness can be expressed as

$\varepsilon_{T}=h v_{T}=h c / \lambda_{T}=4.384 \times 10^{-16} \mathrm{erg} \quad$ (Quantum physics) (12)

where $\varepsilon_{T}=$ quantized energy of the TCP 


$$
\begin{aligned}
&= 4.384 \times 10^{-16} \mathrm{erg}, \\
& v_{T}=\text { frequency of the TCP } \\
&= \varepsilon_{T} / \mathrm{h}=66.12 \times 10^{9} \mathrm{~Hz} .=66.12 \mathrm{GHz}, \\
& \mathrm{h}=\text { Planck's quantum constant }=6.63 \times 10^{-27} \mathrm{erg} \cdot \mathrm{sec}, \\
& \mathrm{c}=\text { free-space velocity of light }=3 \times 10^{10} \mathrm{~cm} / \mathrm{sec}, \\
& \lambda_{T}=\text { wave-length of the } \mathrm{TCP}=0.4537 \mathrm{~cm} .
\end{aligned}
$$

Ultimately this $\mathcal{E}_{T}$ represents universal consciousness.

Thus, $\boldsymbol{\varepsilon}_{T}$ (= consciousness) is found to form a possible gross bridge between the classical physics and quantum physics. Further, Pal [10] has shown that the manifestation of any type of time is simply dependent on the generation of consciousness $\left(=\boldsymbol{\varepsilon}_{T}\right)$. Thus consciousness $\left(=\boldsymbol{E}_{T}\right)$ itself as well as the evolution of time being dependent on the generation of consciousness $\left(=\varepsilon_{T}\right)$ is found to form a possible gross bridge between the classical physics and quantum physics.

\section{Interpretation of the Possible Origin of Matter and Force}

Mermin [11] asked the following questions:

"Are fundamental theories still based on super-positions of states that evolve unitarily, or have the basic principle of quantum mechanics been replaced? If quantum mechanics has survived, have people reached a consensus on the solution to the interpretive problems, or have they simply ceased to view them as problems needing a solution? If quantum mechanics has not survived, has the theory that replaced it clarified these puzzles, or do people find it equally or even more mysterious? Has any progress been made in understanding the nature of conscious experience or how the mind affects the body, and does quantum mechanics or its successor play a fundamental role in that understanding? Did quarks turn out to be elementary or composite? If composite, did the candidates for their constituents turn out to be elementary or composite? Or do we have a better way of looking at these phenomena?"

Physicists determined that underlying quantum fields give birth to elementary particles. Pal [6] explained that thought force $\left(\mathrm{T}_{\mathrm{F}}\right)$ being the primordial quantum field gives birth to TRP that appears to be the origin of all the matter particles. TCP is the carrier of thought force $\left(\mathrm{T}_{\mathrm{F}}\right)$ that, in turn, appears to be the origin of all the fields. TCP thus appears to be the origin of all the field particles.

\section{Phenomenology or Role of Consciousness?}

Phenomenology (from Greek: phainómenon "that which appears"; and lógos "study") is the study of the structure of experience. Phenomenology, in Husserl's conception, is primarily concerned with the systematic reflection on and study of the structures of consciousness and the phenomena that appear in acts of consciousness. Phenomenology (in particle physics) is a branch of particle physics that deals with the application of theory to high-energy experiments.

Why the micro world follows quantum rules and the macro one classical? A quantum description of aggregates of billions of atoms would provide a seamless elegant picture of nature. What prevents such uniformity? Where does the transition take place, and is it sudden?

It has been expressed that TCP is the origin of all the field particles and TRP is the origin of all the matter particles. One TRP (i.e. matter particle $\cong$ fermion) combines with another TRP in the presence of $\mathrm{T}_{\mathrm{F}}$ (micro) that, in turn, is carried by TCP (i.e. field particle $\cong$ boson). These TCP and TRP are the ultimate constituents to constitute a particle in the presence of $\mathrm{T}_{\mathrm{F}}$ (micro) as indicated by Pal [6]. It is possible that there is a critical limit of existence of these associated TRP in the presence of $T_{F}$ (micro). Up to certain extent, these associated TRP in the presence of $T_{F}$ (micro) will obey the quantum rules after which these associated TRP in the presence of $\mathrm{T}_{\mathrm{F}}$ (micro) would have to follow classical rule. Consciousness that, in turn, is the quantized energy $\left(\varepsilon_{T}\right)$ of TCP exerts its inherent functions throughout the universe and this function of consciousness is to be taken into account but usually ignored.

When a particle being constituted by these postulated TCP and TRP in the presence of $T_{F}$ (micro) combines with other particles to form a macroscopic object, the aggregate of particles would be liable to interact with the environment that upsets the delicate balance of quantum states; and the associated combination of these TCP and TRP at a certain level may be responsible to cause this disturbance through the functioning of consciousness. This consciousness, in turn, is the $\varepsilon_{T}$ (= quantized energy of the TCP) and thus makes a cat alive or dead but not in between through the utilization of consciousness. Consciousness thus plays significant roles both in the micro world (that follows quantum rules) and in the macro world (that follows classical rules). It is our plausible opinion that consciousness $\left(=\varepsilon_{T}\right)$ plays significant role to form a possible gross bridge between the classical physics and quantum physics.

Schrödinger (1935) described a thought experiment in which a cat is placed in a box into which poison is released when triggered by a particular quantum event. Schrödinger pointed out that according to the Copenhagen interpretation, the cat would be both dead and alive until the box was opened and the cat observed by a conscious human. Schrödinger cat is the first instance (Thought Experiment) to show a relation between mind (consciousness) and matter in terms of probability (uncertainty). But the present physics has not yet been able to solve the riddle of consciousness. 


\section{Discussion and Conclusion}

\subsection{Discussion}

Consciousness is the quantized energy $\left(\varepsilon_{T}\right)$ of TCP and thus consciousness involves the quantum mechanical activities of these TCP and TRP to which Heisenberg's uncertainty principle is equally applicable. Consciousness will have to play its own role in quantum measurements; and quantum measurements are thus liable to be governed by the uncertainty relations. The term "observation" is nothing but the utilization and involvement of consciousness itself as indicated by Pal et al [2]. Bhaumik [4] pointed out, "In quantum physical experiments, an observer's consciousness is capable of bringing about a particular outcome from the coexisting possibilities inherent in any quantum system".

Pal et al $[1,3]$ explained, "It is possible that the quantum enigma can be explained by accepting the existence of the quantum mechanical activities of these TCP and TRP. Pal et al [3] indicated that these TCP and TRP are the ultimate constituents of any matter as well as any mind in the inherent presence of the thought force $\left(\mathrm{T}_{\mathrm{F}}\right)$ in vitro and thought force $\left(\mathrm{T}_{\mathrm{F}}\right)$ in vivo. The functional state of mind generates consciousness. This consciousness is acting as an inter-linking agent between the animate and inanimate".

Samanta-Laughton [12] expressed, "At a quantum level particles can exist in many states at a time. What brings them out of this superposition is a matter of debate, but according to the Copenhagen interpretation, it is the act of observing a particle that determines what it is. Bohr went further and said that there is no objective reality 'out there'. Things only exist when we observe them. This implies that the whole universe exists only in our consciousness. Although controversial, the Copenhagen interpretation has stood the test of time with its bizarre philosophical implications".

The reigning tenet of quantum mechanics is the uncertainty principle. A consequence of the uncertainty principle is that the presence of an observer or experimenter determines the outcome of the observation or experiment. Simply stated, this means there is no objective reality; you 'create' what you see through the quantum mechanical activities of these TCP and TRP in the presence of consciousness. The quantized energy $\left(\varepsilon_{T}\right)$ of TCP represents universal consciousness. The individual consciousness owes its origin to the universal consciousness created by the same $\varepsilon_{T}$. And these TCP and TRP are the ultimate constituents of any matter as well as any mind in the inherent presence of thought force $\left(\mathrm{T}_{\mathrm{F}}\right)$ in vitro and the thought force $\left(\mathrm{T}_{\mathrm{F}}\right)$ in vivo as it is indicated by $\mathrm{Pal}$ et al $[1$, $3]$.

How could an object's existence depend upon the act of observation? This is due to the fact that an object's existence as well as the act of observation through the prevailing consciousness is totally dependent on the existence of the quantum mechanical activities of these TCP and TRP in the presence of consciousness. The quantized energy $\left(\varepsilon_{T}\right)$ of TCP represents universal consciousness. The individual consciousness owes its origin to the universal consciousness created by the same $\varepsilon_{T}$. And these TCP and TRP are the ultimate constituents of any matter as well as any mind in the inherent presence of thought force $\left(\mathrm{T}_{\mathrm{F}}\right)$ in vitro and the thought force $\left(\mathrm{T}_{\mathrm{F}}\right)$ in vivo as indicated by Pal et al $[1,3]$.

Pal et al $[1,3]$ showed mathematically that the cosmic microwave background radiation (CMBR) temperature is due to the quantized energy $\left(\varepsilon_{T}\right)$ of TCP in the inherent presence of TRP. It is also expressed that the CMBR temperature exists due to the existence of $\mathcal{E}_{T}$ of the TCP radiated from the radiant mass of universe. The existence of the CMBR temperature confirms the existence of TCP in the inherent presence of TRP. The existence of TCP confirms the existence of thought force $\left(\mathrm{T}_{\mathrm{F}}\right)$. Further, this $\mathrm{T}_{\mathrm{F}}$ exerts its functions both in vitro and in vivo. Ultimately this $\mathcal{E}_{T}$ represents universal consciousness. Pal [6] explained the testability for the existence of TCP and TRP along with the Thought force $\left(\mathrm{T}_{\mathrm{F}}\right)$.

\subsection{Conclusion}

Consciousness $\left(=\boldsymbol{\varepsilon}_{T}\right)$ plays significant role to form a possible gross bridge between the classical physics and quantum physics. Pal [10] has also shown that the manifestation of any type of time is simply dependent on the generation of consciousness $\left(=\mathcal{E}_{T}\right)$. Modern scientists may have several important points to consider for the formation of a possible bridge between the classical physics and quantum physics. But it is obvious that the existence of universal consciousness created by the $\mathcal{E}_{T}$ (= quantized energy of TCP) is to be ultimately taken into account. It is expressed here lucidly that consciousness $\left(=\mathcal{E}_{T}\right)$ itself as well as the evolution of time [being dependent on the generation of consciousness $\left.\left(=\mathcal{E}_{T}\right)\right]$ is found to form a possible gross bridge between the classical physics and quantum physics.

It appears that the standard model of physics is to be correctly tuned by ascertaining the constituents of quarks and leptons in the presence of unified field in order to form a possible gross bridge between classical physics and quantum physics.

\section{References}

[1] D. Pal and A.U. De, Physics of consciousness and its model may provide guidelines to solve many scientific problems. Neuroquantology 1: 17-28 (2004)

[2] D. Pal and A.U. De, Consciousness model: Significance of thought-carrying particles and thought-retaining particles in quantum measurement as well as cognitive problem. NeuroQuantology 2: 115-116 (2005) 
[3] D. Pal and A.U. De, The cosmic microwave background radiation temperature signifying the existence of the thought-carrying particle, thought retaining particle and thought force. NeuroQuantology 10: Issue3; 428-442 (September 2012)

[4] M. Bhaumik, Code Name GOD. (Penguin Books India Pvt. Ltd., 11 Community Centre, Panchsheel Park, New Delhi 110 017, India) pp. 132-133; 161-162; 167; 171-72; 177; 185- 186; 89-190; 198 (2006)

[5] V. F. Weisskopf, The Origin of the Universe, The World of Physics, (Simon and Schuster, 1230 Avenue of Americas, New York 10020) 3: 10 (1987)

[6] D. Pal, Existence of thought force and its characteristics. Communicated to American Journal of Modern Physics (AJMP) (2013)

[7] J. A. Wheeler, Beyond the End of Time, The World of Physics, (Simon and Schuster, 1230 Avenue of Americas,
New York 1002), 3: pp. 692 (1987)

[8] D. Pal, Existence of universal consciousness and its characteristics. Accepted for publication in 2014 International Conference on Advanced Education and Management (ICAEM2014) Beijing, China (2013)

[9] S. W. Hawking, A Brief History of Time from the Big Bang to Black Holes. (Bantam Books, 666 fifth Avenue, New York 10103) pp. 165; 136 (1989)

[10] D. Pal, Sense of time and interpretation of the evolution of time. Communicated to American Journal of Modern Physics (AJMP) (2013)

[11] N. David Mermin, What's Wrong with These Questions? Physics Today 11 (February 2001) http://www.physicstoday.org

[12] M. Samanta-Laughton, A Beginner's Guide to Consciousness. The Naked Scientists: Science Radio \& Science Podcasts 2005 